\title{
Carbon Dioxide Capture by Amino Acids Through an Arginine-Arginine Carbamate Ion Pair
}

\author{
Chi-yuan Cheng, ${ }^{\dagger} \S$ Hongwei Shen, ${ }^{\dagger} \S$ Nan-Nan Shen, ${ }^{\ddagger}$ Cristina Castro ${ }^{\dagger}{ }^{\circ}$ Viktor Dubovoy, ${ }^{\dagger}$ Donghui \\ Wu, ${ }^{\dagger}$ Ravi Subramanyan, ${ }^{\dagger}$ Xiao-Ying Huang, ${ }^{\dagger}$ Chloe E. Pitsch, ${ }^{\#}$ Xiaotai Wang, ${ }^{\#}$ Long Pan ${ }^{\dagger},{ }^{*}$ \\ $\dagger$ Colgate-Palmolive Company, 909 River Road, Piscataway, New Jersey 08854, United States \\ $\$$ Key Laboratory of Structural Chemistry, Fujian Institute of Research on the Structure of Matter, \\ Chinese Academy of Sciences, Fuzhou, Fujian 350002, P. R. China \\ \# Department of Chemistry, University of Colorado Denver, Campus Box 194, P.O. Box 173364, \\ Denver, Colorado 80217-3364, United States \\ $\S$ These authors contributed equally \\ *long_pan@colpal.com
}

\section{Reaction of L-arginine and $\mathrm{CO}_{2}$}

L-arginine and $\mathrm{CO}_{2}$ were purchased from Sigma-Aldrich (St. Louis, $\mathrm{MO}$ ) and Airgas (Radnor, PA), respectively, and used directly without further treatment. Deionized water (15 mega-ohm) was generated from Elga Option R PureLab (Woodridge, IL) water purification system. Mettler Toledo Optimax 1001 reactor (Columbus, $\mathrm{OH}$ ) with a $\mathrm{pH}$ monitor and temperature control system was used for the reaction of arginine and $\mathrm{CO}_{2}$. instruments.

Optimax 1001 reactor was first powered on and subsequently $200 \mathrm{~g}$ of deionized water was added. Temperature was set at $70^{\circ} \mathrm{C}$ to start the heating with a stir rate set to $200 \mathrm{RPM}$. For better observation from the front window of the reactor, both the front and back reactor lights were powered on. Once the temperature reached $70{ }^{\circ} \mathrm{C}$, the stirring was turned off and the reactor head assembly was removed. Subsequently, $300 \mathrm{~g}$ of L-arginine was slowly added into the reactor to avoid the arginine powder spreading all over the place. The mixing process started at $200 \mathrm{RPM}$ and then increased to 500 RPM. The temperature was now set to $65^{\circ} \mathrm{C}$. When the temperature reached above $60{ }^{\circ} \mathrm{C}$, the $\mathrm{CO}_{2}$ gas tank was turned on at $50 \mathrm{psi}$ until $\mathrm{pH}$ dropped to 8.5 after the liquid turned clear. The cooling was started until reaching $40{ }^{\circ} \mathrm{C}$. The product was removed from the reactor into a sample jar and used for subsequent measurements.

\section{Preparation of $[\mathrm{Arg}]^{+}[\mathrm{ArgCO}]^{-\cdot} \cdot 2 \mathrm{H}_{2} \mathrm{O}$}

$\mathrm{CO}_{2}$ was pumped into an aqueous arginine suspension $(>60 \mathrm{wt} . \%)$ at $60{ }^{\circ} \mathrm{C}$ to reduce the $\mathrm{pH}$ from 11 to $c a$. 8.5 where a clear solution was formed. The solution was stored in a closed vessel or slowly evaporated at room temperature. Crystalline materials were collected at bottom or inside the vessels. One of the single crystals of $[\mathrm{Arg}]^{+}\left[\mathrm{ArgCO}_{2}\right]^{-} \cdot 2 \mathrm{H}_{2} \mathrm{O}$ (referred to as $\mathrm{CP}-6$ with $\mathrm{CP}$ standing for 
Colgate Palmolive), suitable for single crystal X-ray diffraction, was selected for structural determination.

\section{Powder X-Ray Diffraction (PXRD)}

PXRD of the complex was carried out using a Rigaku D/M-2200T automated diffraction system with $\mathrm{Cu} K \alpha$ irradiation $(\lambda=1.5418 \AA)$. The goniometer was configured in a step-scan mode with $5 \mathrm{~s}$ scans during each $0.02^{\circ}$ step over a range from $\theta=5^{\circ}$ to $\theta=50^{\circ}$.

\section{Single-Crystal X-ray Diffraction (SC-XRD)}

Suitable single crystals were carefully selected under optical microscope and mounted with epoxy on a glass fiber. Data were collected at 175(2) K on a Bruker APEX II diffractometer equipped with a fine focus, $2.0 \mathrm{~kW}$ sealed tube $\mathrm{X}$-ray source $(\mathrm{MoK} \alpha$ radiation, $\lambda=0.71073 \AA$ ). The structure was solved by directed methods and refined by full-matrix least-squares on $F_{2}$ using the SHELX-2014 program package. All non-hydrogen atoms were refined anisotropically. ${ }^{1}$ The $\mathrm{C}-\mathrm{H}$ hydrogen atoms were included in the structural refinement in geometrically idealized positions, while the positions of hydrogen atoms attached to $\mathrm{N}$ and $\mathrm{O}$ atoms were obtained by difference-Fourier synthesis. The $\mathrm{H}$ atoms attached to $\mathrm{N}$ were refined isotropically except for those attached to $\mathrm{N} 8$ which were refined in the riding-model approximation with isotropic displacement parameters set to 1.2 times of the N8. The $\mathrm{H}$ atoms attached to $\mathrm{O}$ were refined with restrained $\mathrm{O}-\mathrm{H}$ of $0.82 \AA$ and their isotropic displacement parameters were set to 1.5 times of the mother $\mathrm{O}$ atoms. The crystallographic data and details of structure-refinement parameters are given in Table S1. Selected bond lengths and angles are listed in Tables S2 and S3.

Table S1. Crystallographic data for CP-6.

\begin{tabular}{|c|c|}
\hline Empirical formula & $\mathrm{C}_{13} \mathrm{H}_{32} \mathrm{~N}_{8} \mathrm{O}_{8}$ \\
\hline Formula Mass & 428.46 \\
\hline Crystal system & Orthorhombic \\
\hline Space group & $P 2_{1} 2_{1} 2_{1}$ \\
\hline$a / \AA$ & $9.3926(8)$ \\
\hline$b / \AA$ & $9.6141(8)$ \\
\hline$c / \AA ̊$ & $21.6527(18)$ \\
\hline$\alpha /^{\circ}$ & 90 \\
\hline$\beta /^{\circ}$ & 90 \\
\hline$\gamma /{ }^{\circ}$ & 90 \\
\hline$V / \AA$ & $1955.3(3)$ \\
\hline$Z$ & 4 \\
\hline$T / \mathrm{K}$ & $175(2)$ \\
\hline$\lambda / \AA$ & 0.71073 \\
\hline Flack parameter & $-0.7(5)$ \\
\hline$F(000)$ & 920 \\
\hline Crystal size $/ \mathrm{mm}$ & $0.20 * 0.14 * 0.12$ \\
\hline$\rho_{\text {catu }} / \mathrm{g} \mathrm{cm}^{3}$ & 1.456 \\
\hline$\mu / \mathrm{mm}$ & 0.120 \\
\hline Measured refls. & 11071 \\
\hline Independent refls. & 4666 \\
\hline
\end{tabular}




\begin{tabular}{|c|c|}
\hline No. of parameters & 327 \\
\hline$R_{u}$ & 0.0282 \\
\hline $\begin{array}{c}R \text { indices }[I>2 \sigma(I)] \\
R, w R 2\end{array}$ & $0.0368,0.0753$ \\
\hline $\begin{array}{c}R \text { indices (all data) } \\
R, w R 2\end{array}$ & $0.0498,0.0811$ \\
\hline GOF & 1.006 \\
\hline Largest diff. peak and hole $/ \AA$ & $0.272,-0.247$ \\
\hline CCDC number & 2090935 \\
\hline
\end{tabular}

[a] $R_{1}=\Sigma\left\|F_{\mathrm{o}}|-| F_{\mathrm{c}}\right\| / \Sigma\left|F_{\mathrm{o}}\right|, w R_{2}=\left[\Sigma w\left(F_{\mathrm{o}}^{2}-F_{\mathrm{c}}^{2}\right)^{2} / \Sigma w\left(F_{\mathrm{o}}^{2}\right)^{2}\right]^{1 / 2}$.

Table S2. Atomic coordinates $\left(\times 10^{4}\right)$ and equivalent isotropic displacement parameters $\left(\AA^{2} \times 10^{3}\right)$ of non-hydrogen atoms for CP-6.

\begin{tabular}{|l|l|l|l|l|l|l|l|l|l|}
\hline Atoms & \multicolumn{1}{|c|}{$\boldsymbol{x}$} & \multicolumn{1}{c|}{$\boldsymbol{y}$} & \multicolumn{1}{c|}{$\boldsymbol{z}$} & $\boldsymbol{U}(\boldsymbol{e q})$ & Atoms & \multicolumn{1}{c|}{$\boldsymbol{x}$} & \multicolumn{1}{c|}{$\boldsymbol{z}$} & $\boldsymbol{z}$ & $\boldsymbol{U}(\boldsymbol{e q})$ \\
\hline $\mathrm{O}(1 \mathrm{~W})$ & $7610(2)$ & $2549(2)$ & $3141(1)$ & $31(1)$ & $\mathrm{N}(7)$ & $3839(2)$ & $8180(2)$ & $3097(1)$ & $19(1)$ \\
\hline $\mathrm{O}(1 \mathrm{~W})$ & $7610(2)$ & $2549(2)$ & $3141(1)$ & $31(1)$ & $\mathrm{N}(8)$ & $808(3)$ & $2288(3)$ & $3083(1)$ & $28(1)$ \\
\hline $\mathrm{O}(2 \mathrm{~W})$ & $7908(2)$ & $5275(2)$ & $3678(1)$ & $28(1)$ & $\mathrm{C}(1)$ & $2193(2)$ & $7771(2)$ & $4544(1)$ & $14(1)$ \\
\hline $\mathrm{O}(1)$ & $6010(2)$ & $1247(2)$ & $5608(1)$ & $24(1)$ & $\mathrm{C}(2)$ & $3213(3)$ & $6088(2)$ & $5284(1)$ & $17(1)$ \\
\hline $\mathrm{O}(2)$ & $4042(2)$ & $349(2)$ & $5198(1)$ & $21(1)$ & $\mathrm{C}(3)$ & $4129(3)$ & $5129(2)$ & $4893(1)$ & $16(1)$ \\
\hline $\mathrm{O}(4)$ & $3414(2)$ & $1187(2)$ & $3409(1)$ & $22(1)$ & $\mathrm{C}(4)$ & $4236(3)$ & $3726(2)$ & $5220(1)$ & $16(1)$ \\
\hline $\mathrm{O}(3)$ & $5615(2)$ & $1050(2)$ & $3810(1)$ & $20(1)$ & $\mathrm{C}(5)$ & $4851(2)$ & $2562(2)$ & $4821(1)$ & $15(1)$ \\
\hline $\mathrm{O}(5)$ & $48(2)$ & $1957(2)$ & $1880(1)$ & $27(1)$ & $\mathrm{C}(7)$ & $4987(3)$ & $1271(2)$ & $5234(1)$ & $16(1)$ \\
\hline $\mathrm{O}(6)$ & $-480(2)$ & $4215(2)$ & $1780(1)$ & $34(1)$ & $\mathrm{C}(8)$ & $4335(3)$ & $1494(2)$ & $3814(1)$ & $16(1)$ \\
\hline $\mathrm{N}(1)$ & $3005(2)$ & $7485(2)$ & $5036(1)$ & $18(1)$ & $\mathrm{C}(9)$ & $4839(2)$ & $7510(3)$ & $3409(1)$ & $16(1)$ \\
\hline $\mathrm{N}(2)$ & $1373(2)$ & $6809(2)$ & $4288(1)$ & $19(1)$ & $\mathrm{C}(10)$ & $4439(3)$ & $5302(3)$ & $2859(1)$ & $20(1)$ \\
\hline $\mathrm{N}(3)$ & $2173(2)$ & $9060(2)$ & $4329(1)$ & $17(1)$ & $\mathrm{C}(11)$ & $2961(3)$ & $4831(2)$ & $3049(1)$ & $18(1)$ \\
\hline $\mathrm{N}(4)$ & $3948(2)$ & $2345(2)$ & $4286(1)$ & $17(1)$ & $\mathrm{C}(12)$ & $2275(3)$ & $4030(3)$ & $2524(1)$ & $20(1)$ \\
\hline $\mathrm{N}(5)$ & $5135(2)$ & $6180(2)$ & $3315(1)$ & $19(1)$ & $\mathrm{C}(13)$ & $776(3)$ & $3530(2)$ & $2670(1)$ & $17(1)$ \\
\hline $\mathrm{N}(6)$ & $5564(3)$ & $8186(2)$ & $3847(1)$ & $22(1)$ & $\mathrm{C}(14)$ & $29(3)$ & $3200(3)$ & $2061(1)$ & $21(1)$ \\
\hline
\end{tabular}

Table S3. Selected bond lengths $(\AA)$ and angles $\left(^{\circ}\right)$ for CP-6.

\begin{tabular}{|l|l|l|l|}
\hline $\mathrm{O}(1 \mathrm{~W})-\mathrm{H}(1 \mathrm{C})$ & $0.834(13)$ & $\mathrm{N}(7)-\mathrm{C}(9)$ & $1.325(3)$ \\
\hline $\mathrm{O}(1 \mathrm{~W})-\mathrm{H}(1 \mathrm{D})$ & $0.830(13)$ & $\mathrm{N}(7)-\mathrm{H}(7 \mathrm{~A})$ & $0.85(3)$ \\
\hline $\mathrm{O}(2 \mathrm{~W})-\mathrm{H}(2 \mathrm{C})$ & $0.829(13)$ & $\mathrm{N}(7)-\mathrm{H}(7 \mathrm{~B})$ & $0.88(3)$ \\
\hline $\mathrm{O}(2 \mathrm{~W})-\mathrm{H}(2 \mathrm{D})$ & $0.831(13)$ & $\mathrm{N}(8)-\mathrm{C}(13)$ & $1.493(3)$ \\
\hline $\mathrm{O}(1)-\mathrm{C}(7)$ & $1.257(3)$ & $\mathrm{N}(8)-\mathrm{H}(8 \mathrm{~A})$ & $0.80(3)$ \\
\hline $\mathrm{O}(2)-\mathrm{C}(7)$ & $1.256(3)$ & $\mathrm{N}(8)-\mathrm{H}(8 \mathrm{~B})$ & $0.92(3)$ \\
\hline $\mathrm{O}(4)-\mathrm{C}(8)$ & $1.266(3)$ & $\mathrm{N}(8)-\mathrm{H}(8 \mathrm{C})$ & $0.91(4)$ \\
\hline $\mathrm{O}(3)-\mathrm{C}(8)$ & $1.276(3)$ & $\mathrm{C}(2)-\mathrm{C}(3)$ & $1.520(3)$ \\
\hline $\mathrm{O}(5)-\mathrm{C}(14)$ & $1.258(3)$ & $\mathrm{C}(2)-\mathrm{H}(2 \mathrm{E})$ & 0.9900 \\
\hline $\mathrm{O}(6)-\mathrm{C}(14)$ & $1.245(3)$ & $\mathrm{C}(2)-\mathrm{H}(2 \mathrm{~F})$ & 0.9900 \\
\hline $\mathrm{N}(1)-\mathrm{C}(1)$ & $1.339(3)$ & $\mathrm{C}(3)-\mathrm{C}(4)$ & $1.527(3)$ \\
\hline $\mathrm{N}(1)-\mathrm{C}(2)$ & $1.460(3)$ & $\mathrm{C}(3)-\mathrm{H}(3 \mathrm{C})$ & 0.9900 \\
\hline $\mathrm{N}(1)-\mathrm{H}(1)$ & $0.86(3)$ & $\mathrm{C}(3)-\mathrm{H}(3 \mathrm{D})$ & 0.9900 \\
\hline
\end{tabular}




\begin{tabular}{|c|c|c|c|}
\hline $\mathrm{N}(2)-\mathrm{C}(1)$ & $1.325(3)$ & $C(4)-C(5)$ & $1.527(3)$ \\
\hline $\mathrm{N}(2)-\mathrm{H}(2 \mathrm{~A})$ & $0.92(3)$ & $\mathrm{C}(4)-\mathrm{H}(4 \mathrm{C})$ & 0.9900 \\
\hline $\mathrm{N}(2)-\mathrm{H}(2 \mathrm{~B})$ & $0.88(3)$ & $\mathrm{C}(4)-\mathrm{H}(4 \mathrm{D})$ & 0.9900 \\
\hline $\mathrm{N}(3)-\mathrm{C}(1)$ & $1.323(3)$ & $C(5)-C(7)$ & $1.536(3)$ \\
\hline $\mathrm{N}(3)-\mathrm{H}(3 \mathrm{~A})$ & $0.84(3)$ & $\mathrm{C}(5)-\mathrm{H}(5 \mathrm{~A})$ & 1.0000 \\
\hline $\mathrm{N}(3)-\mathrm{H}(3 \mathrm{~B})$ & $0.84(3)$ & $\mathrm{C}(10)-\mathrm{C}(11)$ & $1.518(3)$ \\
\hline $\mathrm{N}(4)-\mathrm{C}(8)$ & $1.358(3)$ & $\mathrm{C}(10)-\mathrm{H}(10 \mathrm{~A})$ & 0.9900 \\
\hline $\mathrm{N}(4)-\mathrm{C}(5)$ & 1.451(3) & $\mathrm{C}(10)-\mathrm{H}(10 \mathrm{~B})$ & 0.9900 \\
\hline $\mathrm{N}(4)-\mathrm{H}(4)$ & $0.86(3)$ & $\mathrm{C}(11)-\mathrm{C}(12)$ & $1.517(3)$ \\
\hline $\mathrm{N}(5)-\mathrm{C}(9)$ & 1.324(3) & $\mathrm{C}(11)-\mathrm{H}(11 \mathrm{~A})$ & 0.9900 \\
\hline $\mathrm{N}(5)-\mathrm{C}(10)$ & 1.454(3) & $\mathrm{C}(11)-\mathrm{H}(11 \mathrm{~B})$ & 0.9900 \\
\hline $\mathrm{N}(5)-\mathrm{H}(5)$ & $0.92(3)$ & $\mathrm{C}(12)-\mathrm{C}(13)$ & $1.521(4)$ \\
\hline $\mathrm{N}(6)-\mathrm{C}(9)$ & $1.336(3)$ & $\mathrm{C}(12)-\mathrm{H}(12 \mathrm{~A})$ & 0.9900 \\
\hline $\mathrm{N}(6)-\mathrm{H}(6 \mathrm{~A})$ & $0.89(3)$ & $\mathrm{C}(12)-\mathrm{H}(12 \mathrm{~B})$ & 0.9900 \\
\hline $\mathrm{N}(6)-\mathrm{H}(6 \mathrm{~B})$ & $0.87(4)$ & & \\
\hline $\mathrm{H}(1 \mathrm{C})-\mathrm{O}(1 \mathrm{~W})-\mathrm{H}(1 \mathrm{D})$ & $111(2)$ & $\mathrm{C}(5)-\mathrm{C}(4)-\mathrm{H}(4 \mathrm{C})$ & 108.7 \\
\hline $\mathrm{H}(2 \mathrm{C})-\mathrm{O}(2 \mathrm{~W})-\mathrm{H}(2 \mathrm{D})$ & $113(2)$ & $\mathrm{C}(3)-\mathrm{C}(4)-\mathrm{H}(4 \mathrm{D})$ & 108.7 \\
\hline $\mathrm{C}(1)-\mathrm{N}(1)-\mathrm{C}(2)$ & $123.9(2)$ & $\mathrm{C}(5)-\mathrm{C}(4)-\mathrm{H}(4 \mathrm{D})$ & 108.7 \\
\hline $\mathrm{C}(1)-\mathrm{N}(1)-\mathrm{H}(1)$ & $114(2)$ & $\mathrm{H}(4 \mathrm{C})-\mathrm{C}(4)-\mathrm{H}(4 \mathrm{D})$ & 107.6 \\
\hline $\mathrm{C}(2)-\mathrm{N}(1)-\mathrm{H}(1)$ & $121(2)$ & $\mathrm{N}(4)-\mathrm{C}(5)-\mathrm{C}(4)$ & $109.68(19)$ \\
\hline $\mathrm{C}(1)-\mathrm{N}(2)-\mathrm{H}(2 \mathrm{~A})$ & $123.6(17)$ & $\mathrm{N}(4)-\mathrm{C}(5)-\mathrm{C}(7)$ & 113.41(19) \\
\hline $\mathrm{C}(1)-\mathrm{N}(2)-\mathrm{H}(2 \mathrm{~B})$ & $119(2)$ & $C(4)-C(5)-C(7)$ & 107.11(18) \\
\hline $\mathrm{H}(2 \mathrm{~A})-\mathrm{N}(2)-\mathrm{H}(2 \mathrm{~B})$ & $117(3)$ & $\mathrm{N}(4)-\mathrm{C}(5)-\mathrm{H}(5 \mathrm{~A})$ & 108.8 \\
\hline $\mathrm{C}(1)-\mathrm{N}(3)-\mathrm{H}(3 \mathrm{~A})$ & $118(2)$ & $\mathrm{C}(4)-\mathrm{C}(5)-\mathrm{H}(5 \mathrm{~A})$ & 108.8 \\
\hline $\mathrm{C}(1)-\mathrm{N}(3)-\mathrm{H}(3 \mathrm{~B})$ & $117(2)$ & $\mathrm{C}(7)-\mathrm{C}(5)-\mathrm{H}(5 \mathrm{~A})$ & 108.8 \\
\hline $\mathrm{H}(3 \mathrm{~A})-\mathrm{N}(3)-\mathrm{H}(3 \mathrm{~B})$ & $124(3)$ & $\mathrm{O}(2)-\mathrm{C}(7)-\mathrm{O}(1)$ & $124.6(2)$ \\
\hline $\mathrm{C}(8)-\mathrm{N}(4)-\mathrm{C}(5)$ & 122.1(2) & $\mathrm{O}(2)-\mathrm{C}(7)-\mathrm{C}(5)$ & $118.4(2)$ \\
\hline $\mathrm{C}(8)-\mathrm{N}(4)-\mathrm{H}(4)$ & 115.6(19) & $\mathrm{O}(1)-\mathrm{C}(7)-\mathrm{C}(5)$ & $117.0(2)$ \\
\hline $\mathrm{C}(5)-\mathrm{N}(4)-\mathrm{H}(4)$ & $119.6(18)$ & $\mathrm{O}(4)-\mathrm{C}(8)-\mathrm{O}(3)$ & 124.1(2) \\
\hline $\mathrm{C}(9)-\mathrm{N}(5)-\mathrm{C}(10)$ & $124.8(2)$ & $\mathrm{O}(4)-\mathrm{C}(8)-\mathrm{N}(4)$ & $118.6(2)$ \\
\hline $\mathrm{C}(9)-\mathrm{N}(5)-\mathrm{H}(5)$ & 115.5(19) & $\mathrm{O}(3)-\mathrm{C}(8)-\mathrm{N}(4)$ & $117.3(2)$ \\
\hline $\mathrm{C}(10)-\mathrm{N}(5)-\mathrm{H}(5)$ & $118.6(19)$ & $\mathrm{N}(5)-\mathrm{C}(9)-\mathrm{N}(7)$ & $122.7(2)$ \\
\hline $\mathrm{C}(9)-\mathrm{N}(6)-\mathrm{H}(6 \mathrm{~A})$ & $116.9(19)$ & $\mathrm{N}(5)-\mathrm{C}(9)-\mathrm{N}(6)$ & $118.2(2)$ \\
\hline $\mathrm{C}(9)-\mathrm{N}(6)-\mathrm{H}(6 \mathrm{~B})$ & $116(2)$ & $\mathrm{N}(7)-\mathrm{C}(9)-\mathrm{N}(6)$ & 119.1(2) \\
\hline $\mathrm{H}(6 \mathrm{~A})-\mathrm{N}(6)-\mathrm{H}(6 \mathrm{~B})$ & $123(3)$ & $\mathrm{N}(5)-\mathrm{C}(10)-\mathrm{C}(11)$ & $113.6(2)$ \\
\hline $\mathrm{C}(9)-\mathrm{N}(7)-\mathrm{H}(7 \mathrm{~A})$ & $118.0(18)$ & $\mathrm{N}(5)-\mathrm{C}(10)-\mathrm{H}(10 \mathrm{~A})$ & 108.8 \\
\hline $\mathrm{C}(9)-\mathrm{N}(7)-\mathrm{H}(7 \mathrm{~B})$ & $119.5(19)$ & $\mathrm{C}(11)-\mathrm{C}(10)-\mathrm{H}(10 \mathrm{~A})$ & 108.8 \\
\hline $\mathrm{H}(7 \mathrm{~A})-\mathrm{N}(7)-\mathrm{H}(7 \mathrm{~B})$ & $122(3)$ & $\mathrm{N}(5)-\mathrm{C}(10)-\mathrm{H}(10 \mathrm{~B})$ & 108.8 \\
\hline $\mathrm{C}(13)-\mathrm{N}(8)-\mathrm{H}(8 \mathrm{~A})$ & $108(3)$ & $\mathrm{C}(11)-\mathrm{C}(10)-\mathrm{H}(10 \mathrm{~B})$ & 108.8 \\
\hline $\mathrm{C}(13)-\mathrm{N}(8)-\mathrm{H}(8 \mathrm{~B})$ & $119(2)$ & $\mathrm{H}(10 \mathrm{~A})-\mathrm{C}(10)-\mathrm{H}(10 \mathrm{~B})$ & 107.7 \\
\hline $\mathrm{H}(8 \mathrm{~A})-\mathrm{N}(8)-\mathrm{H}(8 \mathrm{~B})$ & $97(3)$ & $C(12)-C(11)-C(10)$ & 109.7(2) \\
\hline $\mathrm{C}(13)-\mathrm{N}(8)-\mathrm{H}(8 \mathrm{C})$ & $114(2)$ & $\mathrm{C}(12)-\mathrm{C}(11)-\mathrm{H}(11 \mathrm{~A})$ & 109.7 \\
\hline $\mathrm{H}(8 \mathrm{~A})-\mathrm{N}(8)-\mathrm{H}(8 \mathrm{C})$ & $118(3)$ & $\mathrm{C}(10)-\mathrm{C}(11)-\mathrm{H}(11 \mathrm{~A})$ & 109.7 \\
\hline
\end{tabular}




\begin{tabular}{|l|l|l|l|}
\hline $\mathrm{H}(8 \mathrm{~B})-\mathrm{N}(8)-\mathrm{H}(8 \mathrm{C})$ & $100(3)$ & $\mathrm{C}(12)-\mathrm{C}(11)-\mathrm{H}(11 \mathrm{~B})$ & 109.7 \\
\hline $\mathrm{N}(3)-\mathrm{C}(1)-\mathrm{N}(2)$ & $119.9(2)$ & $\mathrm{C}(10)-\mathrm{C}(11)-\mathrm{H}(11 \mathrm{~B})$ & 109.7 \\
\hline $\mathrm{N}(3)-\mathrm{C}(1)-\mathrm{N}(1)$ & $118.7(2)$ & $\mathrm{H}(11 \mathrm{~A})-\mathrm{C}(11)-\mathrm{H}(11 \mathrm{~B})$ & 108.2 \\
\hline $\mathrm{N}(2)-\mathrm{C}(1)-\mathrm{N}(1)$ & $121.3(2)$ & $\mathrm{C}(11)-\mathrm{C}(12)-\mathrm{C}(13)$ & $113.5(2)$ \\
\hline $\mathrm{N}(1)-\mathrm{C}(2)-\mathrm{C}(3)$ & $115.4(2)$ & $\mathrm{C}(11)-\mathrm{C}(12)-\mathrm{H}(12 \mathrm{~A})$ & 108.9 \\
\hline $\mathrm{N}(1)-\mathrm{C}(2)-\mathrm{H}(2 \mathrm{E})$ & 108.4 & $\mathrm{C}(13)-\mathrm{C}(12)-\mathrm{H}(12 \mathrm{~A})$ & 108.9 \\
\hline $\mathrm{C}(3)-\mathrm{C}(2)-\mathrm{H}(2 \mathrm{E})$ & 108.4 & $\mathrm{C}(11)-\mathrm{C}(12)-\mathrm{H}(12 \mathrm{~B})$ & 108.9 \\
\hline $\mathrm{N}(1)-\mathrm{C}(2)-\mathrm{H}(2 \mathrm{~F})$ & 108.4 & $\mathrm{C}(13)-\mathrm{C}(12)-\mathrm{H}(12 \mathrm{~B})$ & 108.9 \\
\hline $\mathrm{C}(3)-\mathrm{C}(2)-\mathrm{H}(2 \mathrm{~F})$ & 108.4 & $\mathrm{H}(12 \mathrm{~A})-\mathrm{C}(12)-\mathrm{H}(12 \mathrm{~B})$ & 107.7 \\
\hline $\mathrm{H}(2 \mathrm{E})-\mathrm{C}(2)-\mathrm{H}(2 \mathrm{~F})$ & 107.5 & $\mathrm{~N}(8)-\mathrm{C}(13)-\mathrm{C}(12)$ & $111.0(2)$ \\
\hline $\mathrm{C}(2)-\mathrm{C}(3)-\mathrm{C}(4)$ & $108.3(2)$ & $\mathrm{N}(8)-\mathrm{C}(13)-\mathrm{C}(14)$ & $111.2(2)$ \\
\hline $\mathrm{C}(2)-\mathrm{C}(3)-\mathrm{H}(3 \mathrm{C})$ & 110.0 & $\mathrm{C}(12)-\mathrm{C}(13)-\mathrm{C}(14)$ & $108.2(2)$ \\
\hline $\mathrm{C}(4)-\mathrm{C}(3)-\mathrm{H}(3 \mathrm{C})$ & 110.0 & $\mathrm{~N}(8)-\mathrm{C}(13)-\mathrm{H}(13)$ & 108.8 \\
\hline $\mathrm{C}(2)-\mathrm{C}(3)-\mathrm{H}(3 \mathrm{D})$ & 110.0 & $\mathrm{C}(12)-\mathrm{C}(13)-\mathrm{H}(13)$ & 108.8 \\
\hline $\mathrm{C}(4)-\mathrm{C}(3)-\mathrm{H}(3 \mathrm{D})$ & 110.0 & $\mathrm{C}(14)-\mathrm{C}(13)-\mathrm{H}(13)$ & 108.8 \\
\hline $\mathrm{H}(3 \mathrm{C})-\mathrm{C}(3)-\mathrm{H}(3 \mathrm{D})$ & 108.4 & $\mathrm{O}(6)-\mathrm{C}(14)-\mathrm{O}(5)$ & $126.7(2)$ \\
\hline $\mathrm{C}(3)-\mathrm{C}(4)-\mathrm{C}(5)$ & $114.15(19)$ & $\mathrm{O}(6)-\mathrm{C}(14)-\mathrm{C}(13)$ & $115.8(2)$ \\
\hline $\mathrm{C}(3)-\mathrm{C}(4)-\mathrm{H}(4 \mathrm{C})$ & 108.7 & $\mathrm{O}(5)-\mathrm{C}(14)-\mathrm{C}(13)$ & $117.4(2)$ \\
\hline
\end{tabular}

Table S4. Hydrogen bonding interactions for CP-6.

\begin{tabular}{|l|l|l|l|l|}
\hline \multicolumn{1}{|c|}{$\mathbf{D}-\mathbf{H} \cdots \mathbf{A}$} & \multicolumn{1}{|c|}{$\mathbf{D}-\mathbf{(} \mathbf{(})$} & $\mathbf{H} \cdots \mathbf{A}(\mathbf{\AA})$ & $\mathbf{D} \cdots \mathbf{A}$ & $\left.<(\mathbf{D H A}) \mathbf{(}^{\circ}\right)$ \\
\hline $\mathrm{O}(1 \mathrm{~W})-\mathrm{H}(1 \mathrm{C}) \cdots \mathrm{O}(3)$ & $0.834(13)$ & $1.947(14)$ & $2.773(3)$ & $171(3)$ \\
\hline $\mathrm{O}(1 \mathrm{~W})-\mathrm{H}(1 \mathrm{D}) \cdots \mathrm{O}(2 \mathrm{~W})$ & $0.830(13)$ & $2.051(13)$ & $2.881(3)$ & $177(3)$ \\
\hline $\mathrm{O}(2 \mathrm{~W})-\mathrm{H}(2 \mathrm{C}) \cdots \mathrm{O}(5) \# 1$ & $0.829(13)$ & $1.962(14)$ & $2.786(3)$ & $173(3)$ \\
\hline $\mathrm{O}(2 \mathrm{~W})-\mathrm{H}(2 \mathrm{D}) \cdots \mathrm{O}(2) \# 2$ & $0.831(13)$ & $1.900(14)$ & $2.724(3)$ & $171(3)$ \\
\hline $\mathrm{N}(1)-\mathrm{H}(1) \cdots \mathrm{O}(2) \# 3$ & $0.86(3)$ & $2.15(3)$ & $2.942(3)$ & $152(3)$ \\
\hline $\mathrm{N}(2)-\mathrm{H}(2 \mathrm{~A}) \cdots \mathrm{O}(1) \# 4$ & $0.92(3)$ & $2.09(3)$ & $2.966(3)$ & $161(2)$ \\
\hline $\mathrm{N}(2)-\mathrm{H}(2 \mathrm{~A}) \cdots \mathrm{O}(2) \# 4$ & $0.92(3)$ & $2.65(3)$ & $3.215(3)$ & $121(2)$ \\
\hline $\mathrm{N}(2)-\mathrm{H}(2 \mathrm{~B}) \cdots \mathrm{O}(5) \# 5$ & $0.88(3)$ & $2.00(3)$ & $2.863(3)$ & $165(3)$ \\
\hline $\mathrm{N}(3)-\mathrm{H}(3 \mathrm{~A}) \cdots \mathrm{O}(2) \# 3$ & $0.84(3)$ & $2.08(3)$ & $2.856(3)$ & $154(3)$ \\
\hline $\mathrm{N}(3)-\mathrm{H}(3 \mathrm{~B}) \cdots \mathrm{O}(6) \# 5$ & $0.84(3)$ & $2.05(4)$ & $2.884(3)$ & $168(3)$ \\
\hline $\mathrm{N}(4)-\mathrm{H}(4) \cdots \mathrm{O}(1) \# 4$ & $0.86(3)$ & $2.26(3)$ & $3.083(3)$ & $161(3)$ \\
\hline $\mathrm{N}(5)-\mathrm{H}(5) \cdots \mathrm{O}(2 \mathrm{~W})$ & $0.92(3)$ & $1.96(3)$ & $2.856(3)$ & $164(3)$ \\
\hline $\mathrm{N}(6)-\mathrm{H}(6 \mathrm{~B}) \cdots \mathrm{N}(1) \# 6$ & $0.87(4)$ & $2.63(4)$ & $3.394(3)$ & $148(3)$ \\
\hline $\mathrm{N}(6)-\mathrm{H}(6 \mathrm{~A}) \cdots \mathrm{O}(3) \# 3$ & $0.89(3)$ & $1.86(3)$ & $2.756(3)$ & $177(3)$ \\
\hline $\mathrm{N}(7)-\mathrm{H}(7 \mathrm{~B}) \cdots \mathrm{O}(4) \# 3$ & $0.88(3)$ & $2.11(3)$ & $2.996(3)$ & $176(3)$ \\
\hline $\mathrm{N}(7)-\mathrm{H}(7 \mathrm{~A}) \cdots \mathrm{O}(1 \mathrm{~W}) \# 1$ & $0.85(3)$ & $2.26(3)$ & $3.066(3)$ & $158(2)$ \\
\hline $\mathrm{N}(8)-\mathrm{H}(8 \mathrm{~A}) \cdots \mathrm{O}(1) \# 4$ & $0.80(3)$ & $2.48(3)$ & $3.169(3)$ & $146(3)$ \\
\hline $\mathrm{N}(8)-\mathrm{H}(8 \mathrm{~B}) \cdots \mathrm{O}(4)$ & $0.92(3)$ & $1.84(4)$ & $2.759(3)$ & $173(3)$ \\
\hline $\mathrm{N}(8)-\mathrm{H}(8 \mathrm{C}) \cdots \mathrm{O}(6) \# 7$ & $0.91(4)$ & $2.30(4)$ & $2.985(3)$ & $132(3)$ \\
\hline $\mathrm{C}(3)-\mathrm{H}(3 \mathrm{C}) \cdots \mathrm{N}(3) \# 6$ & 0.99 & 2.69 & $3.408(3)$ & 130.0 \\
\hline $\mathrm{C}(10)-\mathrm{H}(10 \mathrm{~A}) \cdots \mathrm{N}(7) \# 9$ & 0.99 & 2.51 & $3.326(3)$ & 139.5 \\
\hline $\mathrm{C}(11)-\mathrm{H}(11 \mathrm{~B}) \cdots \mathrm{O}(5) \# 5$ & 0.99 & 2.60 & $3.491(3)$ & 150.3 \\
\hline $\mathrm{C}(13)-\mathrm{H}(13) \cdots \mathrm{O}(5) \# 5$ & 1.00 & 2.62 & $3.522(3)$ & 149.3 \\
\hline
\end{tabular}


Table S5. Classified hydrogen bonding interactions for CP-6.

\begin{tabular}{|c|c|c|c|c|c|}
\hline D-H $\cdots \mathbf{A}$ & D-H ( $(\AA)$ & $\mathbf{H} \cdots \mathbf{A}(\AA)$ & $\mathbf{D} \cdots \mathbf{A}$ & $<$ (DHA) $\left(^{\circ}\right)$ & Note \\
\hline \multicolumn{6}{|c|}{ Inter-layer hydrogen bond formed by A cations (A cations as acceptors) } \\
\hline $\mathrm{O}(2 \mathrm{~W})-\mathrm{H}(2 \mathrm{C}) \cdots \mathrm{O}(5) \# 1$ & $0.829(13)$ & $1.962(14)$ & $2.786(3)$ & $173(3)$ & $\mathrm{A}-\mathrm{H}_{2} \mathrm{O}$ \\
\hline $\mathrm{N}(2)-\mathrm{H}(2 \mathrm{~B}) \cdots \mathrm{O}(5) \# 5$ & $0.88(3)$ & $2.00(3)$ & $2.863(3)$ & $165(3)$ & A-B \\
\hline $\mathrm{N}(3)-\mathrm{H}(3 \mathrm{~B}) \cdots \mathrm{O}(6) \# 5$ & $0.84(3)$ & $2.05(4)$ & $2.884(3)$ & $168(3)$ & A-B \\
\hline \multicolumn{6}{|c|}{ Inter-layer hydrogen bond formed by B anion (B anions as acceptors) } \\
\hline $\mathrm{O}(2 \mathrm{~W})-\mathrm{H}(2 \mathrm{D}) \cdots \mathrm{O}(2) \# 2$ & $0.831(13)$ & $1.900(14)$ & $2.724(3)$ & $171(3)$ & $\mathrm{B}-\mathrm{H}_{2} \mathrm{O}$ \\
\hline $\mathrm{N}(6)-\mathrm{H}(6 \mathrm{~B}) \cdots \mathrm{N}(1) \# 6$ & $0.87(4)$ & $2.63(4)$ & $3.394(3)$ & $148(3)$ & B-A \\
\hline $\mathrm{N}(6)-\mathrm{H}(6 \mathrm{~A}) \cdots \mathrm{O}(3) \# 3$ & $0.89(3)$ & $1.86(3)$ & $2.756(3)$ & $177(3)$ & B-A \\
\hline $\mathrm{N}(7)-\mathrm{H}(7 \mathrm{~B}) \cdots \mathrm{O}(4) \# 3$ & $0.88(3)$ & $2.11(3)$ & $2.996(3)$ & $176(3)$ & B-A \\
\hline $\mathrm{N}(8)-\mathrm{H}(8 \mathrm{~A}) \cdots \mathrm{O}(1) \# 4$ & $0.80(3)$ & $2.48(3)$ & $3.169(3)$ & $146(3)$ & B-A \\
\hline $\mathrm{N}(8)-\mathrm{H}(8 \mathrm{~B}) \cdots \mathrm{O}(4)$ & $0.92(3)$ & $1.84(4)$ & $2.759(3)$ & $173(3)$ & B-A \\
\hline $\mathrm{O}(1 \mathrm{~W})-\mathrm{H}(1 \mathrm{C}) \cdots \mathrm{O}(3)$ & $0.834(13)$ & $1.947(14)$ & $2.773(3)$ & $171(3)$ & $\mathrm{B}-\mathrm{H}_{2} \mathrm{O}$ \\
\hline \multicolumn{6}{|c|}{ Inter-layer hydrogen bond formed by $\mathrm{H}_{2} \mathrm{O}$ molecules $\left(\mathrm{H}_{2} \mathrm{O}\right.$ molecules as acceptors) } \\
\hline $\mathrm{N}(5)-\mathrm{H}(5) \cdots \mathrm{O}(2 \mathrm{~W})$ & $0.92(3)$ & $1.96(3)$ & $2.856(3)$ & $164(3)$ & $\mathrm{H}_{2} \mathrm{O}-\mathrm{A}$ \\
\hline $\mathrm{N}(7)-\mathrm{H}(7 \mathrm{~A}) \cdots \mathrm{O}(1 \mathrm{~W}) \# 1$ & $0.85(3)$ & $2.26(3)$ & $3.066(3)$ & $158(2)$ & $\mathrm{H}_{2} \mathrm{O}-\mathrm{A}$ \\
\hline $\mathrm{O}(1 \mathrm{~W})-\mathrm{H}(1 \mathrm{D}) \cdots \mathrm{O}(2 \mathrm{~W})$ & $0.830(13)$ & $2.051(13)$ & $2.881(3)$ & $177(3)$ & Inter- $\mathrm{H}_{2} \mathrm{O}$ \\
\hline \multicolumn{6}{|c|}{ Intra-B molecule hydrogen bond in Layer $B$} \\
\hline $\mathrm{N}(1)-\mathrm{H}(1) \cdots \mathrm{O}(2) \# 3$ & $0.86(3)$ & $2.15(3)$ & $2.942(3)$ & $152(3)$ & \\
\hline $\mathrm{N}(2)-\mathrm{H}(2 \mathrm{~A}) \cdots \mathrm{O}(1) \# 4$ & $0.92(3)$ & $2.09(3)$ & $2.966(3)$ & $161(2)$ & \\
\hline $\mathrm{N}(2)-\mathrm{H}(2 \mathrm{~A}) \cdots \mathrm{O}(2) \# 4$ & $0.92(3)$ & $2.65(3)$ & $3.215(3)$ & $121(2)$ & \\
\hline $\mathrm{N}(3)-\mathrm{H}(3 \mathrm{~A}) \cdots \mathrm{O}(2) \# 3$ & $0.84(3)$ & $2.08(3)$ & $2.856(3)$ & $154(3)$ & \\
\hline $\mathrm{N}(4)-\mathrm{H}(4) \cdots \mathrm{O}(1) \# 4$ & $0.86(3)$ & $2.26(3)$ & $3.083(3)$ & $161(3)$ & \\
\hline $\mathrm{C}(3)-\mathrm{H}(3 \mathrm{C}) \cdots \mathrm{N}(3) \# 6$ & 0.99 & 2.69 & $3.408(3)$ & 130.0 & \\
\hline \multicolumn{6}{|c|}{ Intra-A molecule hydrogen bond in Layer $A$} \\
\hline $\mathrm{C}(10)-\mathrm{H}(10 \mathrm{~A}) \cdots \mathrm{N}(7) \# 9$ & 0.99 & 2.51 & $3.326(3)$ & 139.5 & \\
\hline $\mathrm{N}(8)-\mathrm{H}(8 \mathrm{C}) \cdots \mathrm{O}(6) \# 7$ & $0.91(4)$ & $2.30(4)$ & $2.985(3)$ & $132(3)$ & \\
\hline $\mathrm{C}(11)-\mathrm{H}(11 \mathrm{~B}) \cdots \mathrm{O}(5) \# 5$ & 0.99 & 2.60 & $3.491(3)$ & 150.3 & \\
\hline $\mathrm{C}(13)-\mathrm{H}(13) \cdots \mathrm{O}(5) \# 5$ & 1.00 & 2.62 & $3.522(3)$ & 149.3 & \\
\hline
\end{tabular}

Symmetry transformations used to generate equivalent atoms: $\# 1-x+1, y+1 / 2,-z+1 / 2 ; \# 2 x+1 / 2,-y+1 / 2,-z+1 ; \# 3 x, y+1, z ; \# 4 x-1 / 2,-y+1 / 2$, $-z+1 ; \# 5-x, y+1 / 2,-z+1 / 2$; \#6 $x+1 / 2,-y+3 / 2,-z+1 ; \# 7-x, y-1 / 2,-z+1 / 2 ; \# 8 x-1 / 2,-y+3 / 2,-z+1 ; \# 9-x+1, y-1 / 2,-z+1 / 2$.

\section{Nuclear Magnetic Resonance (NMR)}

L-arginine and arginine carbamate solutions ( $1 \mathrm{wt} . \%)$ were prepared by dissolving in $\mathrm{D}_{2} \mathrm{O}$ and the $\mathrm{pH}$ was adjusted to 9.0 using $\mathrm{HCl}$ and $\mathrm{NaOH}$. All $\mathrm{NMR}$ spectra were recorded at a temperature of $298 \mathrm{~K}$ using a Bruker DRX500 spectrometer equipped with a double-resonance BBO probe. All experiments with $\mathrm{C}-13$ detection were carried out using a broadband proton decoupling during the 
acquisition and quadrature detection. The $\mathrm{C}-13$ signal detection with ${ }^{1} \mathrm{H}$ selective decoupled was carried out by selectively saturated proton signal corresponding to alpha-proton of arginine carbamate using a soft pulse during the C-13 signal acquisition. The spectra were analyzed using MNova software.

NMR spectra were recorded on a Bruker DMX-500 spectrometer operating at $500.13 \mathrm{MHz} .{ }^{1} \mathrm{H}$ resonance frequency, with a z-field gradient reverse probe and a temperature control held at $298 \mathrm{~K} .{ }^{1} \mathrm{H}$ NMR experiment were recorded with a conventional zg pulse sequence of 5 s recovery delay, accumulating 32 FIDs into $16 \mathrm{~K}$ data points on a $10 \mathrm{ppm}$ spectral width. Fourier transform was computed on $32 \mathrm{~K}$ points. ${ }^{13} \mathrm{C}$ NMR experiment were recorded with a zgig pulse sequence of 5 s recovery delay, accumulating 8k FIDs into $16 \mathrm{~K}$ data point on a $220 \mathrm{ppm}$ spectra width. ${ }^{1} \mathrm{H}-{ }^{13} \mathrm{C}$ HMBC spectra were acquired with a $70 \mathrm{~ms}$ delay for a long-range ${ }^{1} \mathrm{H}-{ }^{13} \mathrm{C}$ coupling selection, with $10 \mathrm{ppm}\left({ }^{1} \mathrm{H}\right)$ and 220 ppm $\left({ }^{13} \mathrm{C}\right)$ spectral width for $2 \mathrm{~h}$. Prior to Fourier transform in magnitude mode, the FIDs were zerofilled in the ${ }^{13} \mathrm{C}$ dimension, and a shifted sine bell function was applied to both dimensions.

Table S6. Peak assignment for the arginine carbamate based on ${ }^{13} \mathrm{C}$ and ${ }^{1} \mathrm{H}$ NMR spectra

\begin{tabular}{|l|l|l|l|}
\hline${ }^{13} \mathbf{C}$ & Chemical shift (ppm) & ${ }^{\mathbf{1}} \mathbf{H}$ & Chemical shift (ppm) \\
\hline$\gamma_{1}$ & 23.62 & $\gamma$ & $0.98-1.11$ \\
\hline$\gamma_{2}$ & 24.31 & $\beta$, & 1.22 \\
\hline$\beta_{1}$ & 27.79 & $\delta$ & 2.55 \\
\hline$\beta_{2}$ & 29.31 & $\alpha$ & 3.05 \\
\hline$\delta_{1}$ & 40.12 & carbamate & 3.26 \\
\hline$\delta_{2}$ & 40.36 & & \\
\hline$\alpha_{1}$ & 53.87 & & \\
\hline$\alpha_{2}$ & 56.05 & & \\
\hline $2_{1}$ & 156.22 & & \\
\hline $2_{2}$ & 156.22 & & \\
\hline & & & \\
\hline 3 & 163.31 & & \\
\hline $1_{1}$ & 175.30 & & \\
\hline $1_{2}$ & 180.78 & & \\
\hline
\end{tabular}

\section{Computational Methods}

Computations were carried out using density functional theory (DFT). Geometries were optimized and characterized by frequency calculations to be energy minima (zero imaginary frequencies) or transition states (only one imaginary frequency) at the M06-2X/BS1 level in aqueous solution with the SMD solvation model, BS1 designating a basis set of 6-31G(d,p) for all atoms..$^{2-3}$ The energies were then refined by M06-2X/BS2//M06-2X/BS1 single-point energy calculations in aqueous solution using the SMD solvation model, BS2 denoting a larger basis set of $6-311++\mathrm{G}(\mathrm{d}, \mathrm{p})$ for all atoms. The refined energies were converted to zero-point energy-corrected free energies at $298.15 \mathrm{~K}$ and 1 atm, using the M06-2X/BS1 harmonic frequencies. All calculations were performed with Gaussian 09. ${ }^{4}$

\section{Hydrolysis experiment for Arginine Carbamate}

To understand how water hydrolyzes the arginine carbamate, we diluted arginine carbamate with water at a series of total arginine concentration. The resulted arginine concentration in wt. \% was quantified by ${ }^{1} \mathrm{H}$ NMR based on the relative peak integrals. 


\section{Additional Figures}

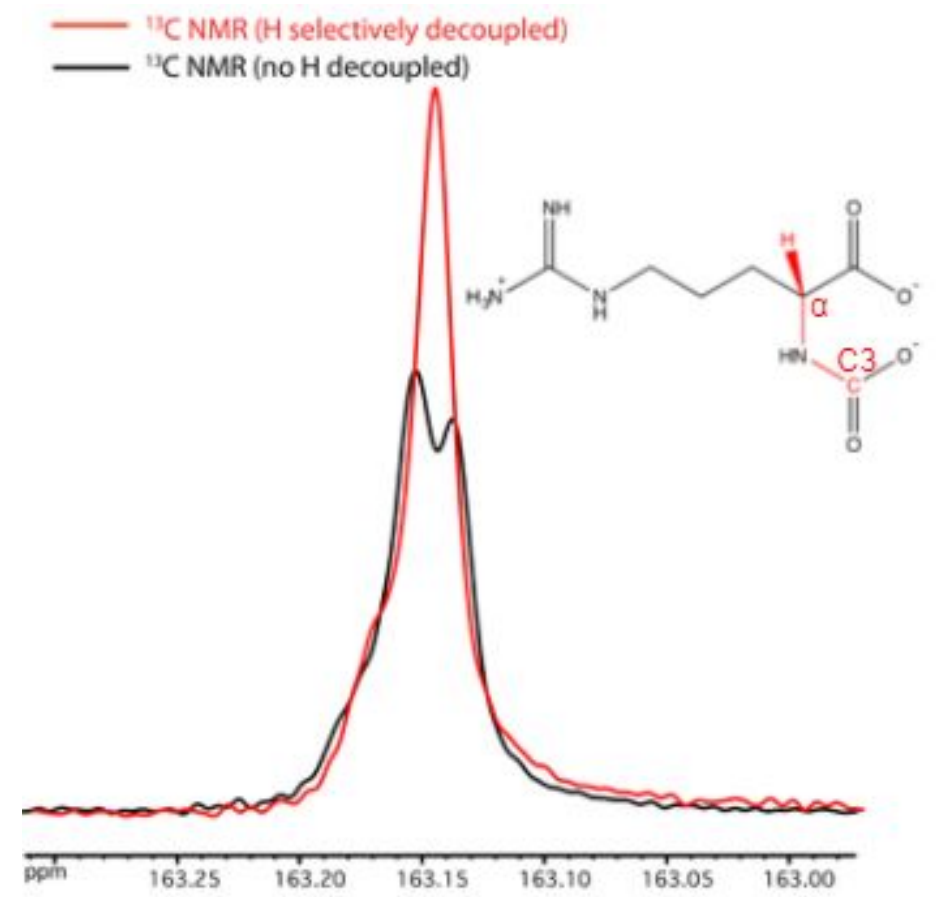

Figure S1. C-13 signal of carbamate carbon with and without proton selectively decoupling

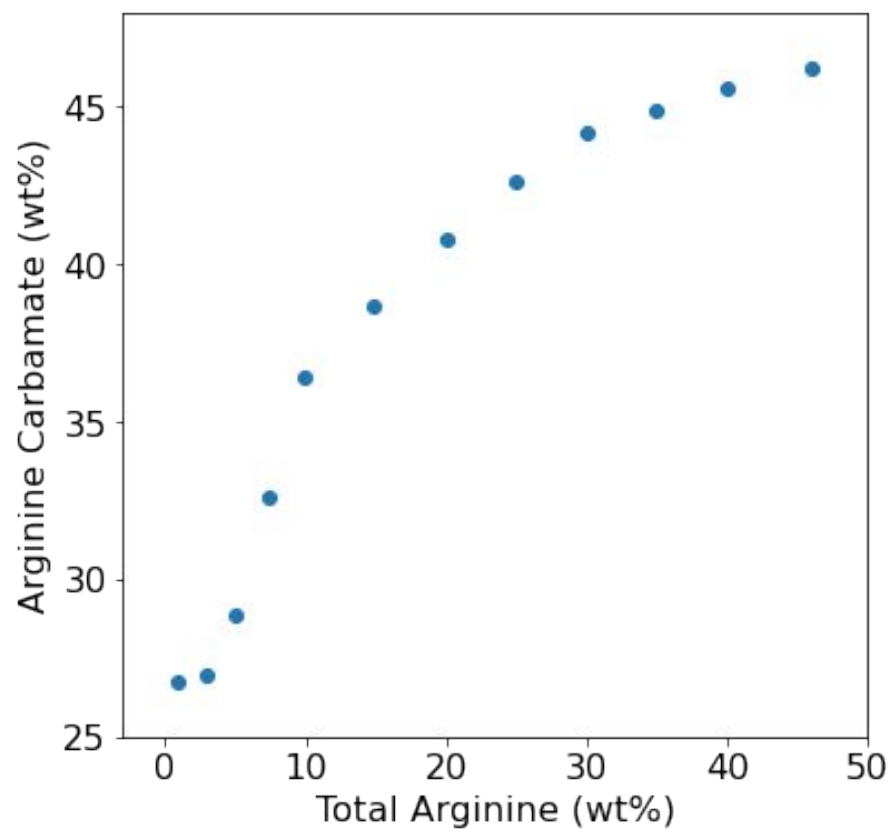

Figure S2. Arginine carbamate concentration as a function of total arginine at $25{ }^{\circ} \mathrm{C}$. Arginine carbamate is hydrolyzed upon diluting the sample with water. The concentrations of arginine carbamate and total arginine were estimated based on the peak integrals of NMR signals. 


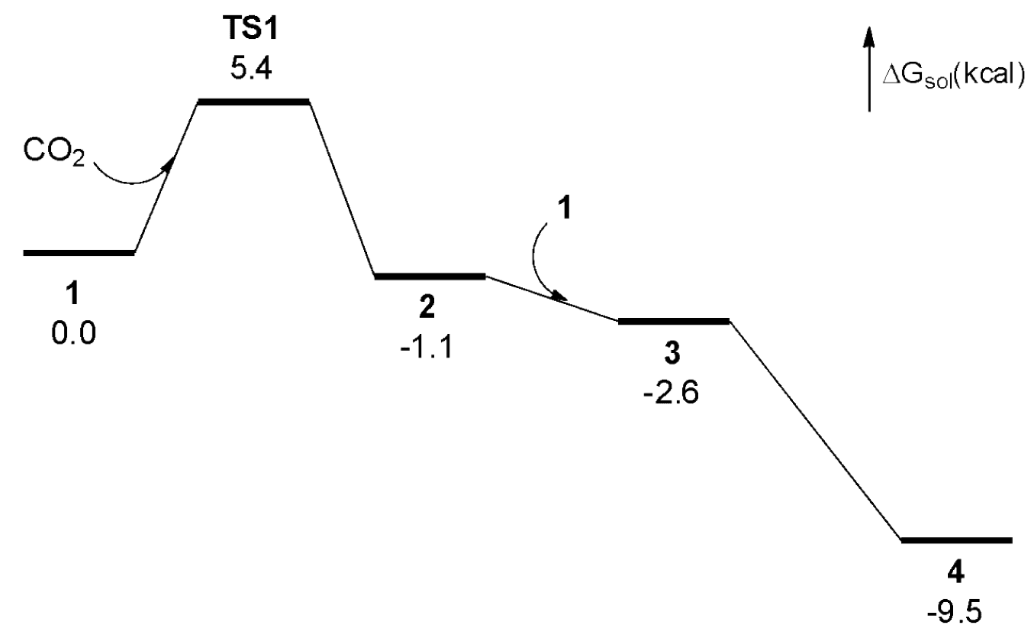

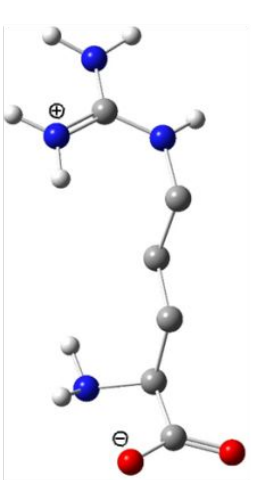

1 (Arg)

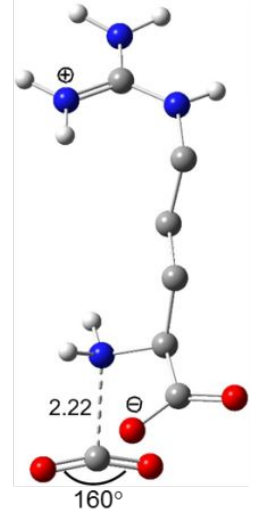

TS1

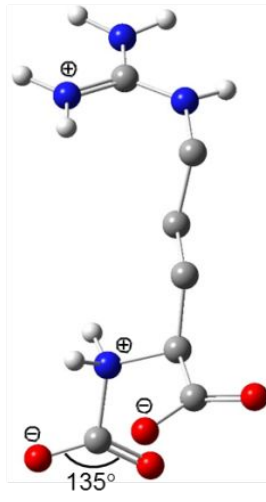

$2\left(\mathrm{ArgCO}_{2}\right)$

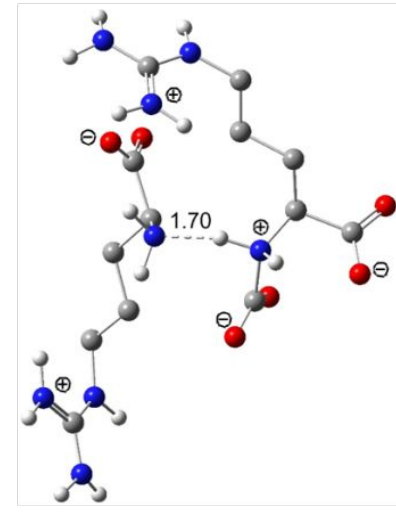

3 (complex)

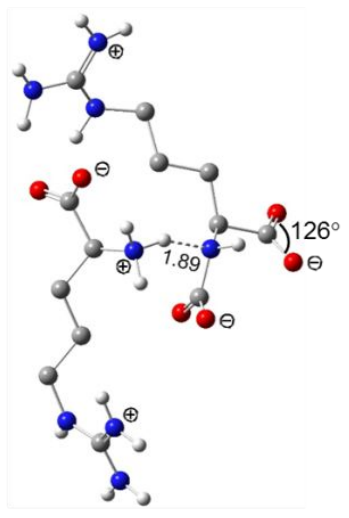

4 (cation-anion pair)

Figure S3. DFT-computed free energy profile for the reaction of $\mathrm{CO}_{2}$ with arginine in aqueous solution to form the arginine-arginine carbamate ion pair $[\mathrm{M} 06-2 \mathrm{X} / 6-311++\mathrm{G}(\mathrm{d}, \mathrm{p}) / \mathrm{SMD}$ (water)//M06-2X/6$31 \mathrm{G}(\mathrm{d}, \mathrm{p}) / \mathrm{SMD}$ (water)], with selected bond distances $(\AA)$ and bond angles. The calculated O-C-O angle $\left(126^{\circ}\right)$ in 4 is consistent with the experimental/crystallographic data 124.6(2); 124.1(2); 126.7(2) (average angle: 125.1). In arginine (1) the $\mathrm{NH}_{2}$ groups far from the carboxylate $\left(\mathrm{COO}^{-}\right)$anion contain a partial positive charge on the $\mathrm{N}$ atoms, thereby being poorer nucleophiles for attacking $\mathrm{CO}_{2}$. This can explain the regioselective carbamate formation involving the $\mathrm{NH}_{2}$ group near the carboxylate anion.

\section{References}

Sheldrick, G. A short history of SHELX. Acta Crystallogr. A 2008, 64 (1), 112-122.

(2) Zhao, Y.; Truhlar, D. G. The M06 suite of density functionals for main group thermochemistry, thermochemical kinetics, noncovalent interactions, excited states, and transition elements: two new functionals and systematic testing of four M06-class functionals and 12 other functionals. Theor. Chem. Acc. 2008, 120 (1), 215-241.

(3) Marenich, A. V.; Cramer, C. J.; Truhlar, D. G. Universal Solvation Model Based on Solute Electron Density and on a Continuum Model of the Solvent Defined by the Bulk Dielectric Constant and Atomic Surface Tensions. J. Phys. Chem. B 2009, 113 (18), 6378-96.

(4) Frisch, M. J.; et al. Gaussian 09, revision D.01, Gaussian, Inc., Wallingford, CT, 2013 